\title{
Was wollen wir?
}

\author{
Rudolf Hammerschmidt
}

Die wachsende Konkurrenz durch privat-gewerbliche Anbieter und die politisch gewollte Entwicklung zu mehr Markt in der Sozialwirtschaft fordert von freigemeinnützigen Hilfsorganisationen, ihre Rolle neu zu definieren und sich zukunftsorientiert zu positionieren. Dass sie wirtschaftlich arbeiten müssen, um am Markt zu bestehen, ist inzwischen anerkannt. Eine Frage ist aber, ob sie dies auch in Zukunft unter den oft einschränkenden gemeinnützigkeitsrechtlichen Rahmenbedingungen tun wollen. Für werteorientierte Träger stellt sich die zentrale Frage nach der individuellen Unternehmensstrategie.

Der Status der Gemeinnützigkeit gilt bei vielen Trägern derzeit noch als eine Art Gütesiegel gegenüber Kunden und Kostenträgern. Doch insbesondere bei den Kostenträgern schwindet dieser Bonus, je mehr Wettbewerb einzieht. Die sozialrechtlichen Privilegien der Freien Wohlfahrtspflege sind in vielen Bereichen längst durch versachlichte Vertragsbeziehungen zwischen Kosten- und Leistungsträgern abgelöst worden. Wenn es um die wirtschaftliche Ausgestaltung ihrer Unternehmen geht, haben privat-gewerbliche Träger insgesamt mehr Freiheiten als gemeinnützige. Die im gemeinnützigen Bereich so schwierigen Diskussionen um Zweckbetriebe und steuerpflichtige wirtschaftliche Geschäftsbetriebe, um das Thema der Gewinnerzielung und Gewinnverwendung etc. müssen nicht geführt werden.

Im Zuge der Europäisierung sollte außerdem perspektivisch eine Streichung der körperschafts- und gewerbesteuerlichen Befreiungen in besonders wettbewerbsintensiven Marktsegmenten einkalkuliert werden. (1) Einige Urteile des Europäischen Gerichtshofes zu wettbewerbsfeindlichen Subventionierungen gibt es bereits. Bisher sind die Auswirkungen noch moderat. Das wird voraussichtlich jedoch nicht so bleiben. Zwar bestehen gemeinnützigkeitsrechtliche Vergünstigungen nicht nur in Deutschland. Zentral für den Bereich sozialer Dienstleistungen ist jedoch die Körperschafts- und Gewerbesteuer.

Vor diesem Hintergrund stellt sich die Frage: Worin besteht der Bonus der Gemeinnützigkeit heute noch? Gemeinnützige Organisationen müssen wirtschaften wie gewerbliche Unternehmen. Aber Geld verdienen dürfen sie nicht. Mit Letzterem sind weniger die steuerrechtlich eingeschränkten Möglichkeiten der Gewinnverwendung gemeint - denn entgegen verbreiteter Meinung dürfen gemeinnützige Unternehmen durchaus Gewinne erwirtschaften. Sie sind lediglich nicht frei in deren Verwendung. Eher wird damit thematisiert, dass es nach wie vor in großen Teilen der Öffentlichkeit verpönt ist, wenn eine

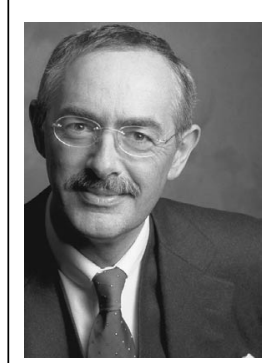

Prof. Dr. Dr. Rudolf Hammerschmidt ist seit 1997 Vorsitzender des Vorstandes der Bank für Sozialwirtschaft AG, Köln/ Berlin. Er ist in zahlreichen Funktionen in der Sozialwirtschaft tätig, u. a. im Präsidialrat der Johanniter-Unfall-Hilfe, im Finanzausschuss des DRK-

Generalsekretariats, im Stiftungsrat der Malteser Stiftung und als Aufsichtsratsvorsitzender der St. Josefshaus Herten Betriebs gGmbH. Internet http://www.sozialbank.de

gemeinnützige Hilfsorganisation Geld verdient. Dass dies mit Blick auf die Ökonomisierung des Sektors und das politisch geforderte professionelle Management in sozialen Organisationen an sich nicht mehr ins Bild passt, kommt kaum jemandem in den Sinn. Allerdings haben sich die gemeinnützigen Organisationen auch mit der Kommunikation dieser widersprüchlichen Anforderungen bisher stark zurückgehalten.

Der Wechsel einer freigemeinnützigen Körperschaft in die Gewerblichkeit könnte von der Öffentlichkeit leicht als Verrat an der Gemeinwohlorientierung aufgefasst werden. Dahinter steckt das Image der Freien Wohlfahrtspflege: Caritas, Diakonie und Co. sind positiv besetzte Marken im Sozialbereich - aller Kritik zum Trotz. Das hohe Ansehen hat viel mit den Gründen zu tun, warum die Organisationen den steuerrechtlichen Status der Gemeinnützigkeit einst erhalten haben: $u$. a. die ausschließliche Ausrichtung auf gemeinnützige, mildtätige und/oder kirchliche Zwecke und das Gebot der Selbstlosigkeit. Diese Voraussetzungen sind nicht mit Blick auf steuerrechtliche Vergünstigungen geschaffen worden, sondern sie sind im Selbstverständnis der Organisationen verankert.

Das Selbstverständnis großer Teile der Freien Wohlfahrtspflege gründet auch heute noch in der selbstlosen Hilfe. Entstanden ist dieses Selbstverständnis jedoch unter völlig anderen Rahmenbedingungen für Soziale Arbeit als den heutigen - nämlich denen, die Ende des 19. und Anfang des 20. Jahrhunderts bei der Gründung der Wohlfahrtsverbände und nach dem Zweiten Weltkrieg in den beiden ersten Dekaden der Bundesrepublik bestanden. Heute jedoch sind sowohl der Armenfürsorge-Gedanke als auch die Subsidiaritäts- und Korporatismusbeziehungen der 1960er und 1970er Jahre weitgehend aus der sozialpolitischen Landschaft verschwunden. 
Die freien Wohlfahrtsverbände - mit Ausnahme des jüdischen Verbandes - sind heute bedeutende Arbeitgeber und Dienstleister. Als Träger wirtschaftlicher Einrichtungen haben sie sich deutlich vom Konzept einer selbstlosen Mildtätigkeit entfernt und sich zu wettbewerblich agierenden Sozialunternehmen gewandelt. Dass dies nicht freiwillig geschehen ist, sondern spätestens seit der Einführung der Sozialen Pflegeversicherung 1994 unter dem politischen Druck einer Neugestaltung des Sozialstaats, ändert wenig am Ergebnis. Die Privatisierung der Nachfrage in der Form, wie sie beispielsweise durch die Einführung des Persönlichen Budgets entsteht, wird den Wandel von der Hilfe- zur Dienstleistung weiter verstärken.

Schon längst konzentrieren sich die Pro- und Kontra-Positionen bezüglich der Gemeinnützigkeit nicht mehr auf die Vertreter der Freien Wohlfahrtspflege auf der einen und der privat-gewerblichen Unternehmen auf der anderen Seite. Die kontroversen Beurteilungen gehen quer durch die verbandlichen Organisationen. Sie betreffen ge-

heblicher Nachholbedarf feststellbar. Es mangelt u. a. an effizienten Controlling- und Steuerungsinstrumentarien, einer aussagekräftigen Finanzplanung und transparenten betriebswirtschaftlichen Kennzahlen. Die Branche könnte wesentlich besser dastehen, würde das brachliegende Potenzial ausgeschöpft.

Viele freigemeinnützige Einrichtungen tun sich nach wie vor schwer mit den wettbewerblichen Rahmenbedingungen auf dem Sozialmarkt. Der Schlüssel zum Verständnis dieser Schwierigkeiten liegt in den traditionellen Funktionen und Organisationszielen der Wohlfahrtsverbände. Sie erfüllen eben nicht nur eine Dienstleistungsfunktion, sondern auch eine Gemeinwohlfunktion. Diese umfasst die Sozialanwaltschaft und das freiwillige Engagement.

Formell werden diese Funktionen in den Satzungen gemeinnütziger Organisationen in der Regel gleich behandelt. Faktisch jedoch hat sich in der Expansionsphase des Sozialstaates in den 1960er und 1970er Jahren eine Hierarchisierung zugunsten der Funktion als Träger professioneller Dienstleistungen durchgesetzt. Diese wurde begünstigt durch das sozialrechtliche Dreiecksverhältnis zwischen Leistungsanbietern, Kostenträgern und Nutzern. Die Funktion der sozialanwaltschaftlichen Interessenvertretung und die Weiterent»Auch gemeinnützige Organisationen dürfen Gewinne machen - sie sind nur nicht frei in deren Verwendung "

gensätzliche Ansichten darüber, was der Verband leisten soll und wie dessen Traditionen bei gleichzeitigen Organisationsveränderungen fortgeführt werden können. (2)

Im Zuge der Ökonomisierung der Sozialwirtschaft wird häufig beklagt, gemeinnützige Organisationen hätten einen unternehmerischen Spagat zu leisten, der kaum zu bewältigen sei. Dieser Spagat besteht in erster Linie darin, mit Blick auf die komplizierten Vorschriften des Steuerrechts die richtige - das heißt gemeinnützigkeitsunschädliche - Anwendung unternehmerischer Gestaltungsmöglichkeiten zu finden. Das erfordert ein differenziertes Know-how. Schon geringfügige Gestaltungsfehler können existenzielle ertrag- und umsatzsteuerliche Folgen nach sich ziehen. Hinzu kommt eine unsichere Planungsgrundlage, die sich aus einem stetigen Wandel und aus grundsätzlichen Defiziten des Steuerrechts ergeben. Dass dieser Spagat leistbar ist, zeigen all diejenigen Träger, die erfolgreich Ausgründungen, Holdingstrukturen, Kooperationen, Fusionen und andere unternehmerischen Ausgestaltungsmöglichkeiten umgesetzt haben. Der Spagat resultiert dagegen nicht - wie oft fälschlich angenommen wird - aus einem grundsätzlichen Widerspruch zwischen gemeinnützigem und wirtschaftlichem Handeln. Im Gegenteil. Es gibt sogar ein Urteil des Bundesfinanzhofes, das besagt, dass wirtschaftliches Handeln auch oberste Maxime für gemeinnütziges Handeln ist. (3)

Das optimale Ausschöpfen von Wirtschaftlichkeitsreserven, der effiziente und effektive Mitteleinsatz um größtmöglichen Nutzen für die gemeinnützigen Zwecke sind zwei der Forderungen, die seit Jahren gestellt werden. Zwar hat sich hier bereits viel getan, aber: Die Schere zwischen denjenigen, die als Gemeinnützige wirtschaftliches Handeln optimiert haben und denjenigen, die von einem durchgängigen Einsatz betriebswirtschaftlicher Instrumente noch weit entfernt sind, klafft innerhalb der Freien Wohlfahrtspflege weit auseinander. Nach wie ist ein er- wicklung der Rolle der ehrenamtlichen Mitarbeiter wurden nicht in gleichem Maße verfolgt. Das Entstehen der Selbsthilfe- und Alternativbewegung in der 1980er Jahren war die gesellschaftliche Reaktion auf diese Entwicklung. (4) Durch die Ökonomisierungsprozesse in den 1990er Jahren wurde die Dominanz der Dienstleistungsfunktion immer größer. $\mathrm{Zu}$ wenig wurde lange Zeit reflektiert, welche Folgen diese Entwicklung für die Identität und damit für die Zukunftsfähigkeit der Wohlfahrtspflege hatte. Dies änderte sich erst Ende der 1990er Jahre, beispielsweise mit dem Trend zur Entwicklung neuer Leitbilder. Hier wurde der Gemeinwohlfunktion wieder eine größere Rolle zugeordnet, zum einen durch eine Rückbesinnung auf das Selbstverständnis, zum anderen als Unterscheidungsmerkmal gegenüber privat-gewerblichen Trägern. Die Herausforderungen für alle Hilfsorganisationen, die gemeinnützig bleiben wollen, liegen heute auf zwei Ebenen:

- Die Gemeinwohlfunktion, insbesondere das ehrenamtliche Engagement, muss strategisch mit der Dienstleistungsfunktion verknüpft werden. Ziel sollte es sein, diese strategische Verknüpfung zu einem Qualitätsstandard zu machen.

- Die Gemeinwohlfunktion muss mit der aktuellen Bürger- und Zivilgesellschaftsdebatte verknüpft werden.

Die strategische Verknüpfung von Gemeinwohlfunktion und Dienstleistungsfunktion lässt sich in der Praxis nur durch die in den letzten Jahren üblich gewordene Ausdifferenzierung der Organisationsstrukturen schaffen: Wirtschaftliche Geschäftsbetriebe werden aus den Vereinsstrukturen ausgegliedert und auf Betriebsgesellschaften übertragen. Damit wird die professionelle Betriebsführung von der ideellen Vereinsführung getrennt. Die Gefahr dabei ist, dass sich die beiden Funktionsbereiche entkoppeln und die Wesensmerkmale der Wohlfahrtspflege nicht mehr sichtbar werden. Ziel sollte jedoch die stra- 
tegische Verzahnung beider Teilsysteme zum Nutzen beider Seiten sein. (5)

Heute haben Freigemeinnützige noch die Chance, ihre Gemeinwohlfunktion als Marketinginstrument einzusetzen, sowohl in der Lobbyarbeit als auch in der internen und externen Öffentlichkeitsarbeit. Denn diese Merkmale unterscheiden sie von Anbietern, denen es ausschließlich um finanzielle Ressourcen und die Sicherung von Marktanteilen geht. Eine Strategie für die Zukunft könnte ein noch aktiverer Gebrauch der sozialanwaltlichen Interessenvertretungsfunktion sein. Das gilt insbesondere dann, wenn diese sich besser als bisher in einer stärkeren Präsenz in den Medien niederschlägt. Die zu erwartende Absenkung des Niveaus der sozialen Sicherung und die daraus folgenden gesellschaftlichen Probleme könnten zu einer Renaissance der sozialanwaltschaftlichen Rolle führen - wenn dies strategisch gewollt ist.

In dem komplexen Feld der Sozialwirtschaft gibt es für traditionelle Hilfsorganisationen vielfältige Optionen, sich zukunftsorientiert aufzustellen. Auf der Seite der Dienstleistungsfunktion stehen unternehmerische Ausgestaltungsmöglichkeiten, die vom effizienten Einsatz betriebswirtschaftlicher Instrumentarien über verschiedenste Outsourcing-Varianten bis hin zur Optimierung von Unternehmensgrößen durch Fusionen und Holdingstrukturen reichen. Auf der Seite der Gemeinwohlfunktionen stehen letztlich ebenfalls unternehmerische Ausgestaltungsmöglichkeiten. Sie betreffen die Nutzung der Gemeinwohlfunktion als Unterscheidungsmerkmal gegenüber privat-gewerblichen Wettbewerbern. Diese

Betrachtungsweise wird einigen Lesern zu pragmatisch sein. Denn damit wird die Gemeinwohlfunktion instrumentalisiert. Es gilt, die Balance zu halten zwischen dem Selbstverständnis und der zusätzlichen Funktion, die Sozialanwaltschaft und Ehrenamt unter den wettbewerblichen Bedingungen einnehmen können.

Die Anforderungen an traditionelle Hilfsorganisationen bleiben auch in den nächsten Jahren immens. Patentrezepte, wie damit umzugehen ist, gibt es nicht. Im Gegenteil: Die Vielfalt der Optionen erfordert maßgeschneiderte Lösungen - nicht, weil das Gemeinnützigkeitsrecht die unternehmerischen Ausgestaltungsmöglichkeiten einschränkt, sondern weil die zentrale Frage die der Unternehmensstrategie ist. Und diese ist in der Regel einmalig, insbesondere für werteorientierte Hilfsorganisationen.

\section{Anmerkungen}

(1) Von Holt, Thomas: Steuerbegünstigungen des sozialen Bereichs, in: Arbeitshandbuch Finanzen für den sozialen Bereich, Kapitel 1.3.

(2) Siehe Boeßenecker, Karl-Heinz: Rahmenbedingungen sozialwirtschaftlicher Prozesse. Zur veränderten Produktion sozialer Dienstleistungen, in: TuP 5/2007.

(3) BFH, Urteil vom 18. Dezember 2002 - I R 60/01.

(4) Siehe Menninger, Oswald: Private und gemeinnützige soziale Dienste. Konkurrenten auf einem gemeinsamen Markt?, in: socialnet.de/Materialien

(5) Ebda.

\section{Ihr Kontakt zur SOZIALwirtschaft ...}

\author{
... für Leserinnen und Leser, die Artikel \\ kommentieren wollen:
}

Gerhard Pfannendörfer, Chefredaktion SOZIALwirtschaft

E-Mail pfannendoerfer@nomos.de

... für Autorinnen und Autoren, die ein Thema vorschlagen wollen:

Gerhard Pfannendörfer,

Chefredaktion SOZIALwirtschaft

E-Mail pfannendoerfer@nomos.de

... für Leserinnen und Leser, die ein Abonnement bestellen wollen:

Aloisia Hohmann, Nomos Verlagsgesellschaft E-Mail hohmann@nomos.de

... für Leserinnen und Leser, die ein Einzelheft bestellen wollen:

Aloisia Hohmann, Nomos Verlagsgesellschaft E-Mail hohmann@nomos.de

... für Organisationen und Agenturen, die eine Anzeige oder eine Beilage schalten wollen:

Kerstin Ternes, Agentur sales friendly E-Mail ternes@sales-friendly.de

... für Leserinnen und Leser, die ein kostenloses Ansichtsexemplar des Informationsdienstes SOZIALwirtschaft aktuell bestellen wollen:

Aloisia Hohmann, Nomos Verlagsgesellschaft E-Mail hohmann@nomos.de

... für Leserinnen und Leser, die sich über das Buchprogramm von Nomos informieren wollen:

Internet http://nomos.de 